\title{
Avaliação antropométrica e de composição corporal de atletas paraolímpicos brasileiros
}

\author{
Sílvio Soares dos Santos ${ }^{1}$ e Fernando José de Sá Pereira Guimarães ${ }^{2}$
}

\section{RESUMO}

Estudos de aspectos relacionados à dimensão, proporção, composição e maturação humanas evidenciaram estreitas relações entre formas e proporções com o movimento humano (Ross e Marfell-Jones apud Petroski, 1999) ${ }^{1}$. Estudos têm demonstrado haver uma relação de algumas medidas antropométricas humanas com risco de doenças metabólicas (assim como o IMC (índice de massa corporal) com o desenvolvimento do diabetes em homens e também em mulheres ${ }^{2-4}$. Não obstante à sua estreita correlação com várias patologias a avaliação antropométrica dos atletas paraolímpicos brasileiros visou identificar o perfil dos diferentes grupos de atletas, descrever suas condições sob o ponto de vista da formação corporal, visando otimizar o seu rendimento atlético durante os Jogos Paraolímpicos de Sydney. Medidas de distribuição da gordura corporal, soma das dobras cutâneas, IMC e RCQ foram alguns dos parâmetros utilizados para se prescrever dietas e treinamentos específicos. Foram detectadas várias alterações nesses itens em vários grupos de atletas. A partir dos dados coletados poder-se-á criar um banco de dados com os parâmetros antropométricos de cada grupo de atletas para futuras comparações.

Palavras-chave: Antropometria. Composição corporal. Esporte paraolímpico.

1. Professor Adjunto II - Faculdade de Educação Física, Universidade Federal de Uberlândia (UFU).

2. Professor da Universidade de Pernambuco (UPE).

Submetido em: 4/4/02

Versão revisada recebida em: 1/5/02

Aceito em: 19/5/02

Endereço para correspondência:

Rua Benjamin Constant, 1.286

38400-678 - Uberlândia, MG

E-mail: silvio@ufu.br

\section{ABSTRACT \\ Anthropometric and body composition evaluation of Bra- zilian paralympic athletes}

A number of studies featuring aspects related to the dimension, proportion, composition, and human maturation show very close relationships between forms and proportions and human movements ${ }^{1}$. It has also been shown that there is a clear relationship between some anthropometric human measurements and the risk of metabolic diseases ${ }^{2}$. Helmrich et al. ${ }^{3}$ have shown the relationship between the IMC (index of corporal mass) and the development of diabetes in men, and Manson et al. ${ }^{4}$ showed similar relationships for women. An anthropometric evaluation of Brazilian paralympic athletes was performed to identify the profile of different groups of athletes and define their status under the light of corporal formation. This study aimed at finding out new methods of optimizing athletic performance during the Sydney 2000 Paralympic Games. Some of the main parameters used to define diets and specific training were the measures of the body fat distribution, total number of cutaneous folds, IMC and RCQ. A number of alterations were detected when comparing those parameters with the different groups of athletes. These data can be used to create a data bank for future studies of anthropometric parameters of disable athletes.

Key words: Anthropometry. Body composition. Paralympic sports.

\section{INTRODUÇÃO}

Ao estudarmos as formas e proporções do homem, torna-se difícil a dissociação delas com o movimento humano. Ross e Marfell-Jones (1991) citado por Petroski (1999)', ao estudarem aspectos relacionados à dimensão, proporção, composição e maturação humanas evidenciaram estreitas relações entre formas e proporções, com o movimento humano.

A cineantropometria ou medida do movimento humano, definida por Petroski $(1995)^{5}$, objetiva dentre outros aspectos avaliar a forma, a dimensão, a proporção, a composição, a maturação e o desenvolvimento do corpo na onto- 
gênese humana em relação ao crescimento, ao desporto, à atividade física e à nutrição.

A cineantropometria está relacionada ainda, segundo Beunen e Borms (1990), citado por Petroski (1999) $)^{1}$, com as características físicas do homem assim como com as medições e avaliações de diferentes aspectos do mesmo, em movimento.

As características físicas podem ser modificadas em função de múltiplos fatores, dentre eles: hábitos quotidianos ${ }^{6}$; hábitos alimentares e quantidade de atividade física (Nóbrega, 1986) ${ }^{7}$; condições socioeconômicas e culturais da família ${ }^{8}$; estado desnutricional ${ }^{9,10}$, ou de elevada nutrição de um povo ${ }^{11}$.

Estudos têm demonstrado haver uma relação de algumas medidas antropométricas humanas com risco de doenças metabólicas, assim como o IMC (índice de massa corporal) com o desenvolvimento do diabetes em homens e também em mulheres ${ }^{2-4}$. As formas andróide e ginóide de distribuição de gordura corporal estão relacionadas com várias doenças. A primeira relaciona-se com doenças crônico-degenerativas (hipertensão, cânceres etc.) assim como várias outras medidas antropométricas ao desenvolvimento de desordens metabólicas e coronarianas (Van Itallie apud Guimarães, 1999) ${ }^{12}$.

Não obstante à sua estreita correlação com várias patologias a avaliação antropométrica dos atletas paraolímpicos brasileiros não visou identificar neles qualquer relação de morbidade. Ela visou sim e sobretudo, identificar o perfil dos diferentes grupos de atletas participantes daqueles Jogos, descrever suas condições sob o ponto de vista da formação corporal além de subsidiar ações de prescrição no campo da nutrição e do treinamento, visando otimizar o seu rendimento atlético durante os Jogos Paraolímpicos de Sydney.

\section{METODOLOGIA}

A antropometria foi dividida em nove grandes grupos de medidas, que visavam descrever o perfil morfológico de cada atleta. Foram utilizados os seguintes instrumentos para medição: antropômetro para as medidas de largura, altura, comprimento e profundidade; compasso de Lange, para medição de dobras cutâneas; balança digital para a medida da massa corporal; uma prancha de madeira com balança, para localização do centro de gravidade corporal (CG); fita métrica antropométrica com precisão de $0,1 \mathrm{~cm}$, para as medidas de circunferência; compasso de pontas
TABELA 1

Quadro classificatório da cineantropometria

Cineantropometria

\begin{tabular}{|c|c|c|c|}
\hline Identificação & Especificação & Aplicação & Relevância \\
\hline Cineantropometria & $\begin{array}{c}\text { Para o estudo } \\
\text { do homem }\end{array}$ & $\begin{array}{l}\text { Para ajudar o } \\
\text { entendimento }\end{array}$ & $\begin{array}{c}\text { Com aplicações } \\
\text { para: }\end{array}$ \\
\hline $\begin{array}{l}\text { Mensuração do } \\
\text { movimento } \\
\text { humano }\end{array}$ & $\begin{array}{l}\text { Tamanho } \\
\text { Forma } \\
\text { Proporção } \\
\text { Composição } \\
\text { Maturação } \\
\text { Função }\end{array}$ & $\begin{array}{c}\text { Crescimento } \\
\text { Exercício } \\
\text { Performance } \\
\text { Estado nutricional }\end{array}$ & $\begin{array}{l}\text { Educação } \\
\text { Medicina } \\
\text { Governo } \\
\text { Trabalho } \\
\text { Esportes }\end{array}$ \\
\hline
\end{tabular}

Adaptado de Ross e Marfell-Jones (1991) por Petroski (1999)

rombudas para medir medidas de profundidade e de largura; e finalmente o estadiômetro, para a medida de estatura. Dessa forma, as medidas foram assim conduzidas:

Medida da massa corporal (Petroski, 1999) ${ }^{\mathbf{1}}$ - tomada em balança digital com precisão de 50gr;

Medidas de alturas (Petroski, 1999) ${ }^{1}$ - feitas com o antropômetro em posição ortostática com unidade em centímetro.

- Altura do centro de gravidade corporal; Altura sentado; Altura dos ombros direito e esquerdo; Altura dos quadris direito e esquerdo; Altura dos joelhos direito e esquerdo.

Medidas de profundidade (Petroski, 1999) ${ }^{1}$ - foram conduzidas no plano sagital com precisão de milímetros, com o compasso de pontas rombas e pontas retas.

- Profundidade da cintura; Profundidade das nádegas; Profundidade do tronco; Largura máxima sagital do tronco.

Medidas de largura ${ }^{1}$ - foram conduzidas no plano frontal com o compasso antropométrico de pontas retas com precisão de milímetros, mantido paralelamente ao solo.

- Largura do tronco; Largura dos quadris; Envergadura.

Medida de comprimento ${ }^{1}$ - medida com paquímetro antropométrico com precisão de milímetros.

- Comprimento dos pés.

Medidas de circunferência ${ }^{1}$ - foram feitas com uma fita antropométrica com precisão de milímetros.

- Circunferência do peito; Circunferência da cintura; Circunferência do abdome; Circunferência do quadril; Circunferência braço; Circunferência coxa; Circunferência panturrilha.

Dobras cutâneas - medida com o compasso de Lange segundo protocolos padronizados ${ }^{1}$.

- Dobra bicipital; Dobra axilar; Dobra tricipital; Dobra subescapular; Dobra abdominal; Dobra supra ilíaca; Dobra da coxa; Dobra da panturrilha. 
Estatura $^{1}$ - medida em posição ortostática do chão ao topo da cabeça com o olhar dirigido para frente com antropômetro com precisão de mm.

Densidade corporal ${ }^{13}$ - medida através de pesagem hidrostática.

\section{RESULTADOS}

As tabelas seguintes mostram o resumo dos resultados das medidas antropométricas das equipes paraolímpicas brasileiras. São mostradas a soma das medidas centrais (S. central) em porcentagem, a soma das medidas periféricas (S. periférica) em porcentagem, a soma das oito dobras (S. Dobras), a estatura, o índice de massa corporal (IMC), que é relação entre a massa corporal e a estatura ao quadrado, e a idade média de cada atleta.

Ao final de cada tabela observamos ainda a média, o desviopadrão, os valores mínimos e máximos e o coeficiente de variabilidade da amostra quando a mesma for maior que três elementos. Algumas medidas colocadas em vermelho e itálico na tabela, destacam valores acima da média do grupo.

A tabela 2 mostra os resultados da equipe paraolímpica brasileira de futebol. O coeficiente de variabilidade foi especialmente alto na soma das dobras demonstrando uma heterogeneidade entre os atletas nesse parâmetro. Os atletas mostraram uma predominância de percentual de gordura na parte central do corpo (60\%). Quatro dos atletas apresentaram em relação ao parâmetro "soma das dobras", índices superiores aos da média do grupo $(\sim 50 \%)$. Cinco dos atletas mostraram índices superiores aos da média do grupo $(\sim 8 \%)$ para o "IMC", sendo quatro deles, aqueles mesmos atle- tas que já apresentaram índices acima da média no parâmetro soma das dobras.

A tabela 3 mostra os resultados com os valores médios, o desvio-padrão, os valores mínimos e máximos e o coeficiente de variabilidade das medidas antropométricas da equipe de basquete paraolímpica.

\begin{tabular}{|c|c|c|c|c|c|c|}
\hline \multicolumn{7}{|c|}{$\begin{array}{c}\text { TABELA } 2 \\
\text { Medidas antropométricas da equipe de futebol }\end{array}$} \\
\hline Atletas & $\begin{array}{l}\text { S. central } \\
(\%)\end{array}$ & $\begin{array}{l}\text { S. periférica } \\
(\%)\end{array}$ & $\begin{array}{l}\text { S. dobras } \\
(\mathrm{mm})\end{array}$ & $\begin{array}{l}\text { Estatura } \\
(\mathbf{c m})\end{array}$ & $\underset{\left(\mathrm{kg} / \mathrm{m}^{2}\right)}{\mathrm{IMC}}$ & $\begin{array}{l}\text { Idade } \\
\text { (anos) }\end{array}$ \\
\hline M.F. & 66,41 & 33,59 & 128 & 178 & 22,03 & 18,7 \\
\hline M.L. & 55,77 & 44,23 & 52 & 176 & 20,5 & 23,4 \\
\hline L. & 65,29 & 34,71 & 121 & 174 & 23,78 & 24,1 \\
\hline F. & 66,67 & 33,33 & 48 & 176,5 & 20,22 & 34,5 \\
\hline R. & 53,06 & 46,94 & 49 & 176,5 & 19,32 & 20,9 \\
\hline J. & 55,32 & 44,68 & 47 & 182 & 18,42 & 18,9 \\
\hline A.C. & 51,39 & 48,61 & 72 & 176 & 20,47 & 25 \\
\hline M. & 62,5 & 37,5 & 48 & 178 & 20,26 & 21,4 \\
\hline J.A. & 61,79 & 38,21 & 123 & 174 & 24,11 & 27,6 \\
\hline D. & 63,64 & 36,36 & 88 & 187 & 21,85 & 35,3 \\
\hline M.W. & 58,82 & 41,18 & 68 & 177 & 22,41 & 20,1 \\
\hline Média & 60,06 & 39,94 & 76,73 & 177,73 & 21,22 & 24,54 \\
\hline DP & 5,47 & 5,47 & 32,97 & 3,76 & 1,78 & 5,79 \\
\hline Mín. & 51,39 & 33,33 & 47,00 & 174,00 & 18,42 & 18,70 \\
\hline Máx. & 66,67 & 48,61 & 128,00 & 187,00 & 24,11 & 35,30 \\
\hline C. Variab. & 9,12 & 13,71 & 42,97 & 2,12 & 8,41 & 23,58 \\
\hline
\end{tabular}

TABELA 3

Medidas antropométricas da equipe de basquete

\begin{tabular}{lcccccc}
\hline Atletas & $\begin{array}{c}\text { S. central } \\
(\%)\end{array}$ & $\begin{array}{c}\text { S. periférica } \\
(\%)\end{array}$ & $\begin{array}{c}\text { S. dobras } \\
(\mathbf{m m})\end{array}$ & $\begin{array}{c}\text { Estatura } \\
(\mathbf{c m})\end{array}$ & $\begin{array}{c}\text { IMC } \\
\left(\mathbf{k g} / \mathbf{m}^{2}\right)\end{array}$ & $\begin{array}{c}\text { Idade } \\
\text { (anos) }\end{array}$ \\
C.S. & 57,83 & 42,17 & 83 & 187,5 & 20,65 & 18,7 \\
L. & 58,04 & 41,96 & 112 & 189,5 & 24,9 & 26,7 \\
J. & 62,11 & 37,89 & 95 & 182 & 25,51 & 27,5 \\
N. & 68,84 & 31,16 & 138 & 200 & 24,93 & 27,7 \\
I. & 53,23 & 46,77 & 124 & 205,5 & 22,69 & 28 \\
A.L. & 56 & 44 & 125 & 174,5 & 25,65 & 23,7 \\
G. & 60,71 & 39,29 & 56 & 170,5 & 18,85 & 21,7 \\
R.R. & 69,92 & 30,08 & 123 & 180,5 & 22,41 & 24 \\
D.B. & 57,14 & 42,86 & 63 & 178 & 20,64 & 17,5 \\
A.C. & 61,19 & 38,81 & 67 & 183 & 20,36 & 22 \\
A.O. & 54,69 & 45,31 & 64 & 180 & 20,31 & 17,2 \\
A.M. & 56,72 & 43,28 & 134 & 182 & 21,62 & 31,1 \\
Média & $\mathbf{5 9 , 7 0}$ & $\mathbf{4 0 , 3 0}$ & $\mathbf{9 8 , 6 7}$ & $\mathbf{1 8 4 , 4 2}$ & $\mathbf{2 2 , 3 8}$ & $\mathbf{2 3 , 8 2}$ \\
DP & $\mathbf{5 , 2 2}$ & $\mathbf{5 , 2 2}$ & $\mathbf{3 0 , 8 3}$ & $\mathbf{1 0 , 0 2}$ & $\mathbf{2 , 3 5}$ & $\mathbf{4 , 5 2}$ \\
Mín. & $\mathbf{5 3 , 2 3}$ & $\mathbf{3 0 , 0 8}$ & $\mathbf{5 6 , 0 0}$ & $\mathbf{1 7 0 , 5 0}$ & $\mathbf{1 8 , 8 5}$ & $\mathbf{1 7 , 2 0}$ \\
Máx. & $\mathbf{6 9 , 9 2}$ & $\mathbf{4 6 , 7 7}$ & $\mathbf{1 3 8 , 0 0}$ & $\mathbf{2 0 5 , 5 0}$ & $\mathbf{2 5 , 6 5}$ & $\mathbf{3 1 , 1 0}$ \\
C. Variab. & $\mathbf{8 , 7 4}$ & $\mathbf{1 2 , 9 5}$ & $\mathbf{3 1 , 2 5}$ & $\mathbf{5 , 4 3}$ & $\mathbf{1 0 , 5 1}$ & $\mathbf{1 9 , 0 0}$ \\
\hline
\end{tabular}


O coeficiente de variabilidade foi alto na soma das dobras demonstrando uma heterogeneidade entre os atletas nesse parâmetro. Os atletas mostraram uma predominância de percentual de gordura na parte central do corpo $(59,7 \%)$. A estatura média da equipe $(184 \mathrm{~cm})$ se comparada com equipes olímpicas dessa modalidade se mostra relativamente baixa. Seis dos atletas apresentaram em relação ao parâmetro "soma das dobras", índices superiores aos da média do grupo ( 26\%). Seis dos atletas mostraram índices superiores aos da média do grupo ( 9\%) para o "IMC", sendo cinco deles, aqueles mesmos atletas que já apresentaram índices acima da média no parâmetro soma das dobras.

A tabela 4 mostra os resultados das medidas antropométricas dos corredores da equipe paraolímpica de atletismo. Nela encontram-se a média, o desvio-padrão, os valores mínimos e máximos e o coeficiente de variabilidade dessa equipe.

O coeficiente de variabilidade não foi alto para a soma das dobras mostrando portanto uma heterogeneidade relativa entre os atletas nesse parâmetro $(17,3 \%)$. Os atletas mostraram uma predominância de percentual de gordura na parte central do corpo $(62,6 \%)$. A estatura média da equipe foi de $172,8 \mathrm{~cm}$ com uma variabilidade bastante baixa $(\sim 4,7 \%)$. Quatro dos atletas apresentaram em relação ao parâmetro "soma das dobras", índices superiores aos da média do grupo $(\sim 12 \%)$. Apenas dois dos atletas mostraram índices superiores aos da média do grupo ( 9\%) para o "IMC", estando esses atletas entre aqueles que apresentaram índices acima da média no parâmetro soma das dobras.

A tabela 5 mostra os valores das medidas antropométricas das corredoras da equipe paraolímpica de atletismo. Não foi possível estabelecer qualquer comparação ou mesmo determinar seus valores médios e o coeficiente de variabilidade da amostra devido ao pequeno número de atletas (dois). É interessante notar que ambas as atletas participam de provas curtas mas apresentam uma inversão na distribuição da gordura corporal. Outro aspecto interessante é a grande diferença mostrada na soma das dobras ( 79\%), suge- 
rindo algum excesso de tecido adiposo em uma das atletas não obstante o seu IMC estar dentro de parâmetros razoáveis. $\mathrm{O}$ percentual de gordura estimado das atletas foi de $20,43 \%$ para a atleta M.J. e de $12,33 \%$ para a atleta A.S., uma diferença de quase $8 \%$. Apesar da atleta A.S. apresentar uma massa corporal menor $(52,4 \mathrm{~kg})$ em relação à atleta M.J. (55,4kg), a primeira mostra uma quantidade de massa magra estimada maior $(45,94 \mathrm{~kg})$ quando comparada com a outra atleta $(44,08 \mathrm{~kg})$. Isso sugere que a primeira apresenta uma melhor preparação corporal que a segunda.

A tabela 6 mostra os resultados das medidas antropométricas das arremessadoras da equipe paraolímpica de atletismo.

O coeficiente de variabilidade não foi alto para a soma das dobras mostrando portanto uma heterogeneidade relativa entre as atletas nesse parâmetro (11,9\%). As atletas mostraram uma predominância de percentual de gordura na parte central do corpo $(62,3 \%)$. A estatura média da equipe $(141,8 \mathrm{~cm})$ com uma variabilidade percentual de $30,5 \%$ reflete a condição das atletas envolvidas, em especial uma com amputação de ambos os membros inferiores). Em relação ao parâmetro "soma das dobras", o índice absoluto alcançado foi muito alto (277,3mm). Se levarmos ainda em consideração que duas das atletas apresentam amputações de membros inferiores, o quadro geral fica ainda pior. O "IMC" desse grupo foi muito alto em especial o da atleta com dupla amputação de membros inferiores $(94,3 \mathrm{~kg} /$ $\mathrm{m}^{2}$ ). O percentual de gordura não pode ser estimado para as atletas amputadas por não existir protocolos diferenciados para esse grupo de atletas. Outro índice calculado foi o da relação cintura-quadril (RCQ), que variou entre 0,87 a 0,94 nesse grupo, demonstrando assim um elevado risco devido à alta concentração de tecido adiposo na região central baixa do corpo.

Pode-se então perceber pela soma de dobras cutâneas, pelo IMC e pela RCQ que o grupo não se encontra em condições corporais ótimas para a prática da atividade atlética de elite.

A tabela 7 mostra os resultados do único arremessador da equipe paraolímpica brasileira. Os parâmetros IMC e RCQ encon- tram-se dentro de limites aceitáveis quando comparados com os índices populacionais. A distribuição da gordura corporal mostra-se uma consonância com os demais atletas das outras modalidades apesar da existência de grandes diferenças estruturais entre a natureza de algumas delas.

A tabela 8 mostra os resultados das medidas antropométricas da equipe de judô paraolímpica.

$\mathrm{O}$ coeficiente de variabilidade foi muito alto na soma das dobras $(50,44 \%)$ demonstrando uma heterogeneidade entre os atletas nesse parâmetro. Isso se deveu principalmente ao tratamento em conjunto desses atletas que pertencem à diferentes categorias. Os atletas mostraram uma predominância de percentual de gordura na parte central do corpo $(71,2 \%)$. Dois dos atletas apresentaram em relação ao parâmetro "soma das dobras", índices superiores aos da média do grupo de $\sim 54 \%$. Três dos atletas mostraram índices bastante elevados para o "IMC" e que desses três atletas dois mostram índices de RCQ bastante preocupantes.

A tabela 9 mostra os resultados das medidas antropométricas da equipe masculina paraolímpica de tênis de mesa.

O coeficiente de variabilidade foi alto para a soma das dobras mostrando portanto uma predominância de percentual de gordura na parte central do corpo (59,3\%). O "IMC" desse grupo não mostrou-se muito alto apenas um atleta apresentou um índice elevado com $36,1 \mathrm{~kg} / \mathrm{m}^{2}$ assim como

TABELA 7

Medidas antropométricas do atleta de atletismo (arremessos)

\begin{tabular}{ccccccc}
\hline Atleta & $\begin{array}{c}\text { S. central } \\
(\%)\end{array}$ & $\begin{array}{c}\text { S. periférica } \\
(\%)\end{array}$ & $\begin{array}{c}\text { S. dobras } \\
(\mathbf{m m})\end{array}$ & $\begin{array}{c}\text { Estatura } \\
(\mathbf{c m})\end{array}$ & $\begin{array}{c}\text { IMC } \\
\left(\mathbf{k g} / \mathbf{m}^{2}\right)\end{array}$ & $\begin{array}{c}\text { Idade } \\
(\mathbf{a n o s})\end{array}$ \\
A. & 55,37 & 44,63 & 121 & 186 & 25,09 & 28,4 \\
\hline
\end{tabular}

TABELA 8

Medidas antropométricas da equipe de judô masculino

\begin{tabular}{lcccccc}
\hline Atletas & $\begin{array}{c}\text { S. central } \\
(\%)\end{array}$ & $\begin{array}{c}\text { S. periférica } \\
(\%)\end{array}$ & $\begin{array}{c}\text { S. dobras } \\
(\mathbf{m m})\end{array}$ & $\begin{array}{c}\text { Estatura } \\
(\mathbf{c m})\end{array}$ & $\begin{array}{c}\text { IMC } \\
\left(\mathbf{k g} / \mathbf{m}^{2}\right)\end{array}$ & $\begin{array}{r}\text { Idade } \\
\text { (anos) }\end{array}$ \\
H. & 64,18 & 35,82 & 67 & 162 & 24,01 & 23 \\
A. & 64,18 & 35,82 & 67 & 169,5 & 23,11 & 27,8 \\
T. & 75 & 25 & 96 & 180 & 29,38 & 29,8 \\
D. & 72,19 & 27,81 & 187 & 170,5 & 33,37 & 27,2 \\
L. & 80,33 & 19,67 & 183 & 176 & 36,67 & 38,3 \\
Média & $\mathbf{7 1 , 1 8}$ & $\mathbf{2 8 , 8 2}$ & $\mathbf{1 2 0 , 0 0}$ & $\mathbf{1 7 1 , 6 0}$ & $\mathbf{2 9 , 3 1}$ & $\mathbf{2 9 , 2 2}$ \\
DP & $\mathbf{7 , 0 2}$ & $\mathbf{7 , 0 2}$ & $\mathbf{6 0 , 5 2}$ & $\mathbf{6 , 8 5}$ & $\mathbf{5 , 8 6}$ & $\mathbf{5 , 6 5}$ \\
Mín. & $\mathbf{6 4 , 1 8}$ & $\mathbf{1 9 , 6 7}$ & $\mathbf{6 7 , 0 0}$ & $\mathbf{1 6 2 , 0 0}$ & $\mathbf{2 3 , 1 1}$ & $\mathbf{2 3 , 0 0}$ \\
Máx. & $\mathbf{8 0 , 3 3}$ & $\mathbf{3 5 , 8 2}$ & $\mathbf{1 8 7 , 0 0}$ & $\mathbf{1 8 0 , 0 0}$ & $\mathbf{3 6 , 6 7}$ & $\mathbf{3 8 , 3 0}$ \\
C. Variab. & $\mathbf{9 , 8 7}$ & $\mathbf{2 4 , 3 7}$ & $\mathbf{5 0 , 4 4}$ & $\mathbf{3 , 9 9}$ & $\mathbf{1 9 , 9 8}$ & $\mathbf{1 9 , 3 3}$ \\
\hline
\end{tabular}


para a RCQ $(0,99)$. O percentual de gordura não pode ser estimado para dois atletas por não existirem protocolos diferenciados para o cálculo do percentual de gordura para ambos os casos. A RCQ para esses mesmos dois atletas também não pode ser determinada devido à dificuldade para a tomada das medidas além da inexistência de um protocolo específico para a tomada das mesmas.

A tabela 10 mostra os dados da atleta de tênis de mesa, que apresenta elevados índices de IMC, RCQ e percentual de gordura. Tais índices são alarmantes se considerarmos se tratar de uma atleta.
A tabela 11 apresenta os resultados dos atletas de ciclismo. Ambos os atletas apresentam boa condição em relação aos índices IMC e RCQ. No entanto existe grande diferença entre eles no parâmetro soma das dobras $(48 \mathrm{~mm})$, o que os diferencia bastante em relação à quantidade percentual de gordura em seus corpos.

A tabela 12 apresenta os resultados dos atletas de halterofilismo. Ambos os atletas apresentam elevados índices IMC e RCQ. Existe uma boa chance da RCQ ter sido elevada nos atletas devido à presença de uma atrofia muscular observada à partir dos seus quadris. O IMC para ambos os atletas mostrou-se também elevado, sugerindo que deva haver uma redução da massa corporal de ambos, ou seja, da quantidade de gordura corporal em excesso.

A tabela 13 mostra os resultados da atleta de halterofilismo da equipe paraolímpica brasileira. Quanto aos parâmetros IMC e RCQ a atleta encontra-se dentro da normalidade da população. Há que se ressaltar o fato de a distribuição da gordura corporal apresentar uma maior porcentagem na periferia $(\sim 52,5)$ ao invés de na parte central do corpo. Tal distribuição não era esperada se considerarmos que o maior trabalho ou esforço físico nessa modalidade é desenvolvido nos membros superiores e a quantidade de gordura nos membros inferiores não é substancialmente diferente da quantidade encontrada nos atletas dessa modalidade com características físicas semelhantes.

A tabela 14 mostra os resultados das medidas antropométricas dos nadadores da equipe paraolímpica brasileira.

O coeficiente de variabilidade foi alto para a soma das dobras mostrando portanto uma heterogeneidade relativa entre os atletas nesse parâmetro $(41,8 \%)$. Os atletas mostraram uma predominância de percentual de gordura na parte central

\begin{tabular}{lcccccc}
\hline Atletas & $\begin{array}{c}\text { S. central } \\
(\%)\end{array}$ & $\begin{array}{c}\text { S. periférica } \\
(\%)\end{array}$ & $\begin{array}{c}\text { S. dobras } \\
(\mathbf{m m})\end{array}$ & $\begin{array}{c}\text { Estatura } \\
(\mathbf{c m})\end{array}$ & $\begin{array}{c}\text { IMC } \\
\left(\mathbf{k g} / \mathbf{m}^{2}\right)\end{array}$ & $\begin{array}{c}\text { Idade } \\
\text { (anos) }\end{array}$ \\
A. & 77,94 & 22,06 & 136 & 168,5 & 29,23 & 30,5 \\
J.E. & 68 & 32 & 200 & 156,5 & 28,17 & 33,3 \\
\hline
\end{tabular}


do corpo $(58,3 \%)$. A estatura média da equipe foi de $164,7 \mathrm{~cm}$ com uma variabilidade bastante baixa $(\sim 8 \%)$. Seis dos atletas apresentaram em relação ao parâmetro "soma das dobras", índices superiores aos da média do grupo te visual, um que tenha seqüela de pólio e outro tetraplegia por acidente automobilístico.

A comparação inter-grupos, pelo mesmo motivo, tornase uma tarefa ainda mais difícil se considerarmos agora $(\sim 42 \%)$. Sete dos atletas mostraram índices superiores aos da média do grupo $(\sim 11 \%)$ para o "IMC", estando quatro desses atletas entre aqueles que apresentaram índices acima da média no parâmetro soma das dobras. Os altos coeficientes de variabilidade encontrados nesse grupo de nadadores deve-se à grande variedade de deficiências encontradas nesse grupo.

A tabela 15 mostra o grupo de nadadoras paraolímpicas brasileiras. A variabilidade foi elevada apenas no parâmetro idade $(28,2 \%)$. Nos demais parâmetros a variabilidade foi baixa, ou seja, inferiores a $7,02 \%$. Nesse grupo encontramos uma atleta deficiente visual e duas com paraplegia. O IMC encontra-se dentro de padrões normais, quando comparados com dados tabelados para a população normal. É interessante notar que a distribuição da gordura para duas atletas foi maior na periferia que na parte central, apesar do grande trabalho desenvolvido pelos membros superiores e inferiores nessa modalidade.

\section{CONCLUSÃO}

Torna-se extremamente difícil uma análise geral e conclusiva acerca de todos os parâmetros avaliados em cada atleta, devido principalmente à grande variedade de tipos de deficiências encontradas entre eles.

Do ponto de vista da ciência é uma tarefa muito complicada fazer comparações intragrupo quando esse grupo é constituído por exemplo por um deficien-

\begin{tabular}{ccccccr}
\hline \multicolumn{7}{c}{ TABELA 13 } \\
Medidas antropométricas da atleta de halterofilismo \\
\hline Atleta & $\begin{array}{c}\text { S. central } \\
(\%)\end{array}$ & $\begin{array}{c}\text { S. periférica } \\
(\%)\end{array}$ & $\begin{array}{c}\text { S. dobras } \\
(\mathbf{m m})\end{array}$ & $\begin{array}{c}\text { Estatura } \\
(\mathbf{c m})\end{array}$ & $\begin{array}{c}\text { IMC } \\
\left(\mathbf{k g} / \mathbf{m}^{2}\right)\end{array}$ & $\begin{array}{c}\text { Idade } \\
\text { (anos) }\end{array}$ \\
T. & 47,53 & 52,47 & 162 & 153,5 & 23,26 & 28,8 \\
\hline
\end{tabular}

TABELA 14

Medidas antropométricas da equipe de natação masculina

\begin{tabular}{|c|c|c|c|c|c|c|}
\hline Atletas & $\begin{array}{c}\text { S. central } \\
(\%)\end{array}$ & $\begin{array}{c}\text { S. periférica } \\
(\%)\end{array}$ & $\begin{array}{l}\text { S. dobras } \\
(\mathrm{mm})\end{array}$ & $\begin{array}{l}\text { Estatura } \\
\text { (cm) }\end{array}$ & $\begin{array}{c}\text { IMC } \\
\left(\mathrm{kg} / \mathrm{m}^{2}\right)\end{array}$ & $\begin{array}{l}\text { Idade } \\
\text { (anos) }\end{array}$ \\
\hline L.S. & 31,33 & 68,67 & 166 & 130 & 31,3 & 18,8 \\
\hline G. & 56,25 & 43,75 & 144 & 155,5 & 23,99 & 24 \\
\hline D. & 61,45 & 38,55 & 83 & 172 & 22,51 & 23,3 \\
\hline M.B. & 60,24 & 39,76 & 83 & 174 & 21,37 & 18,6 \\
\hline J.A. & 65 & 35 & 160 & 177,5 & 24,66 & 32,9 \\
\hline F.A. & 49,06 & 50,94 & 53 & 162 & 18,33 & 34,4 \\
\hline A.G. & 65,63 & 34,38 & 96 & 169 & 22,16 & 27,1 \\
\hline P. & 65,63 & 34,38 & 128 & 170,3 & 24,17 & 27,3 \\
\hline J. & 60,13 & 39,87 & 153 & 159 & 20,45 & 33,8 \\
\hline F.M. & 60 & 40 & 55 & 178 & 21,62 & 25,2 \\
\hline C.F. & 51,79 & 48,21 & 56 & 173 & 22,25 & 21,4 \\
\hline I. & 79,75 & 20,25 & 79 & 161 & 19,21 & 27,8 \\
\hline G. & 58 & 42 & 50 & 150 & 15,64 & 27,9 \\
\hline A.G.P. & 51,3 & 48,7 & 115 & 174,5 & 20,53 & 38,9 \\
\hline Média & 58,25 & 41,75 & 101,50 & 164,70 & 22,01 & 27,24 \\
\hline DP & 10,89 & 10,89 & 42,41 & 13,17 & 3,61 & 6,03 \\
\hline Mín. & 31,33 & 20,25 & 50,00 & 130,00 & 15,64 & 18,60 \\
\hline Máx. & 79,75 & 68,67 & 166,00 & 178,00 & 31,30 & 38,90 \\
\hline C. Variab. & 18,69 & 26,08 & 41,78 & 7,99 & 16,40 & 22,15 \\
\hline
\end{tabular}

TABELA 15

Medidas antropométricas da equipe de natação feminina

\begin{tabular}{lcccccr}
\hline Atletas & $\begin{array}{c}\text { S. central } \\
(\%)\end{array}$ & $\begin{array}{c}\text { S. periférica } \\
(\%)\end{array}$ & $\begin{array}{c}\text { S. dobras } \\
(\mathbf{m m})\end{array}$ & $\begin{array}{c}\text { Estatura } \\
(\mathbf{c m})\end{array}$ & $\begin{array}{c}\text { IMC } \\
\left(\mathbf{k g} / \mathbf{m}^{2}\right)\end{array}$ & $\begin{array}{r}\text { Idade } \\
\text { (anos) }\end{array}$ \\
F. & 47,22 & 52,78 & 144 & 156 & 21,41 & 19,6 \\
C. & 53,73 & 46,27 & 161 & 152,5 & 19,65 & 34,5 \\
R. & 48,28 & 51,72 & 145 & 148 & 19,27 & 25,6 \\
Média & $\mathbf{4 9 , 7 4}$ & $\mathbf{5 0 , 2 6}$ & $\mathbf{1 5 0 , 0 0}$ & $\mathbf{1 5 2 , 1 7}$ & $\mathbf{2 0 , 1 1}$ & $\mathbf{2 6 , 5 7}$ \\
DP & $\mathbf{3 , 4 9}$ & $\mathbf{3 , 4 9}$ & $\mathbf{9 , 5 4}$ & $\mathbf{4 , 0 1}$ & $\mathbf{1 , 1 4}$ & $\mathbf{7 , 5 0}$ \\
Mín. & $\mathbf{4 7 , 2 2}$ & $\mathbf{4 6 , 2 7}$ & $\mathbf{1 4 4 , 0 0}$ & $\mathbf{1 4 8 , 0 0}$ & $\mathbf{1 9 , 2 7}$ & $\mathbf{1 9 , 6 0}$ \\
Máx. & $\mathbf{5 3 , 7 3}$ & $\mathbf{5 2 , 7 8}$ & $\mathbf{1 6 1 , 0 0}$ & $\mathbf{1 5 6 , 0 0}$ & $\mathbf{2 1 , 4 1}$ & $\mathbf{3 4 , 5 0}$ \\
C. Variab. & $\mathbf{7 , 0 2}$ & $\mathbf{6 , 9 5}$ & $\mathbf{6 , 3 6}$ & $\mathbf{2 , 6 4}$ & $\mathbf{5 , 6 8}$ & $\mathbf{2 8 , 2 2}$ \\
\hline
\end{tabular}


que a comparação deve ser feita para modalidades diferentes.

No entanto, à partir de agora teremos já como referencia esses parâmetros, que até então eram completamente desconhecidos da maioria daqueles que militam nessa área. À partir deles e para os próximos anos já teremos condições de comparar e avaliar os atletas quando da formação de nossas equipes paraolímpicas.

A criação de um banco de dados que contenha o maior número possível de informações acerca dos atletas paraolímpicos brasileiros será sem dúvida o nosso próximo e grande desafio. O primeiro passo já foi dado. A alimentação contínua desse banco de dados poderá nos dar maiores subsídios e com isso maior confiança nas tomadas de deci-

\section{REFERÊNCIAS}

1. Petroski EL. Antropometria: técnicas e padronizações. Porto Alegre: Pallotti, 1999.

2. Guimarães FJ, Souza OF, Pires Neto CS, et al. Classificação do padrão da gordura corporal em crianças. Anais do II Encontro Internacional para Estudos da Criança. Santa Maria, 1996:35.

3. Helmrich SP, Ragland DR, Leung RW, Paffenbarger RS. Physical activity and reduced occurrence of non-insulin-dependent diabetes mellitus. N Engl J Med 1991;325:147-52.

4. Manson JE, Rimm EB, Stampfer MJ, et al. Physical activity and incidence of non-insulin-dependent diabetes mellitus in women. Lancet 1991; 338:774-8.

5. Malina RM. Growth and development. Minneapolis: Burges Publishing Co., 1975

6. Nóbrega G. Desnutrição: intra-uterina e pós-natal. São Paulo: Panamed Editorial, 1986. são acerca de qual atleta selecionar, qual tipo de treinamento ministrar, qual o melhor método para se avaliar determinada síndrome etc.

Entendo esse trabalho de avaliação como pioneiro e de extrema importância para o país, na medida que mostra, quantifica e avalia uma classe de atletas tão singulares e especiais.

\section{AGRADECIMENTOS}

- Comitê Paraolímpico Brasileiro (CPB)

- Secretaria Nacional de Esportes

- Rede Cenesp/Unifesp

- Associação Fundo de Incentivo à Psicofarmacologia (Afip)

- Universidade Federal de São Paulo (Unifesp)

7. Marcondes E, et al. Desenvolvimento neuropsicomotor da criança desnutrida. Psiquiatria 1969;4:175-219.

8. Lohman TG. Advances in body composition assessment: current issues in exercise science. Monograph 3. Champaign, Illinois: Human Kinetics, 1992.

9. Dietz WH. Obesity, weight control, and eating disorders. In: Cheung LWY, Richmond JB, editors. Child health, nutrition and physical activity. Champaign, Illinois: Human Kinetics, 1995:155-66.

10. Guimarães FJ. Distribuição da gordura corporal: relação com o perfil lipídico e glicêmico de coronariopatas atendidos no hospital universitário Oswaldo Cruz, na cidade de Recife. Tese de Doutorado. Santa Maria, 1999.

11. Pollock ML, Wilmore JH. Exercícios na saúde e na doença. Rio de Janeiro: Editora Médica e Científica, 1993. 